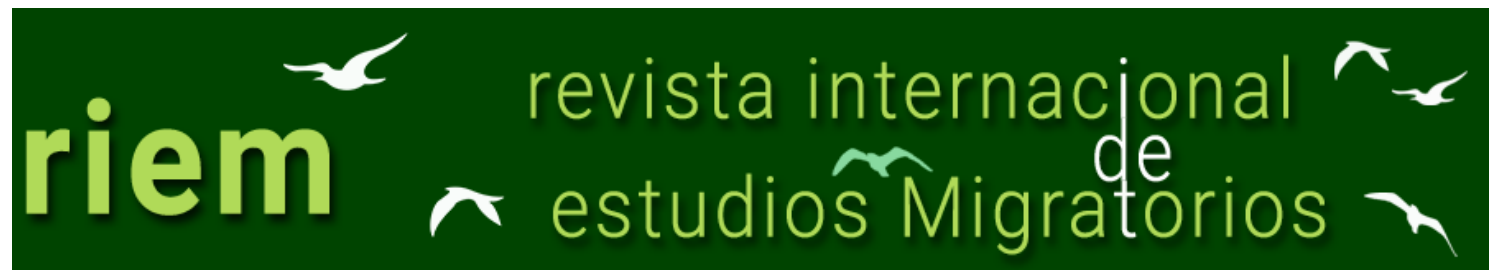

ISSN: $2173-1950$

\title{
Validación de la estructura teórica de los ámbitos socioculturales del Modelo Ampliado de Aculturación Relativa
}

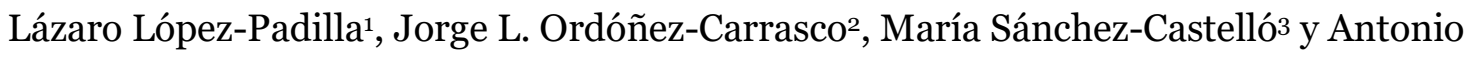
José Rojas-Tejada4*

Resumen: El Modelo Ampliado de Aculturación Relativa (MAAR) propone distintos ámbitos de la realidad sociocultural (político, bienestar social, laboral, económico, social, familiar, religioso y de valores) que pueden clasificarse en ámbitos públicos-periféricos o privados-centrales según el grado de preferencia en mantener la cultura de origen y adoptar la cultura de acogida. El objetivo de este trabajo consiste en aportar evidencias empíricas del modelo teórico de los ámbitos de aculturación propuestos por el MAAR a partir de las medidas de adopción y de mantenimiento utilizando los mapas de variables propios de los modelos logísticos de Rasch en distintos grupos (autóctonos que evalúan a rumanos y a ecuatorianos). El trabajo se realizó aplicando un cuestionario a 922 personas españolas (493 evaluaron a personas de origen ecuatoriano y 499 de origen rumano) mediante muestreo aleatorio multietápico. En la primera etapa, un equipo de expertos seleccionó, los municipios con mayores porcentajes de inmigrantes (al menos el $5 \%$ del censo tenía que ser inmigrantes y, de éstos, al menos el $2 \%$ eran rumanos/ecuatorianos). En la segunda etapa se estratificó, con una asignación

1 Departamento de Psicología, Universidad de Almería, Almería. España. lazarolp18@gmail.com

2 Centro de Investigación para el Estudio de las Migraciones y las Relaciones Interculturales (CEMyRI) y Departamento de Psicología, Universidad de Almería, Almería. España. joc657@ual.es

3 Centro de Investigación para el Estudio de las Migraciones y las Relaciones Interculturales (CEMyRI) y Departamento de Psicología, Universidad de Almería, Almería. España.msc943@ual.es

4 Centro de Investigación para el Estudio de las Migraciones y las Relaciones Interculturales (CEMyRI) y Departamento de Psicología, Universidad de Almería, Almería. España. arojas@ual.es

* Autor de correspondencia 
proporcional por sexo y edad y, por último, se seleccionaron rutas aleatorias en cada municipio. El error de muestreo se estimó en $\pm 4,3 \%$. Los resultados obtenidos muestran evidencias de validez empírica del MAAR. En los ámbitos privados, los autóctonos prefieren que los inmigrantes mantengan más de su cultura de origen, así como una preferencia menor por la adopción de la cultura de acogida. En los ámbitos considerados públicos, los autóctonos prefieren que los inmigrantes adopten más la cultura de acogida y menos su cultura de origen. Este estudio avala las predicciones teóricas propuestas por el MAAR referentes al orden de los ámbitos socioculturales, tanto en las preferencias de mantenimiento como de adopción, en ambos grupos de autóctonos.

Palabras Clave: aculturación, modelo ampliado de aculturación relativa, modelo de Rasch, teoría de respuesta a los ítems. 


\title{
Validation of the theoretical structure of the socio- cultural domains of the Relative Acculturation Extended Model
}

\begin{abstract}
The Relative Acculturation Extended Model of (RAEM) contemplates different areas of socio-cultural reality (political, social welfare, labor, economic, social, family, religious and values), being able to be classified in public-peripheral or privatecentral spheres according to the degree of preference in maintaining the culture of origin and adopting the host culture. The aim of this paper is to provide empirical evidence of the theoretical model of the areas of acculturation proposed by the RAEM based on the adoption and maintenance measures applying a model based on the item response theory (IRT) and using variables maps by Rasch's logistic models in different groups (natives people evaluating Romanians and Ecuadorians). The work was carried out by applying a questionnaire to 922 Spanish people (493 evaluated Ecuadorians and 499 evaluated Romanians) by a multi-stage sampling. In the first stage, a team of experts selected, from the national census data, the municipalities with the highest percentages of immigrants (at least $5 \%$ of the census had to be immigrants and of these, at least $2 \%$ were Romanian/Ecuadorian). In the second stage it was stratified, with a proportional allocation by sex and age and, finally, random routes were selected in each municipality. The sampling error was estimated at $\pm 4.3 \%$. Results show empirical evidence about the validity of the RAEM. Private areas the natives prefer immigrants to maintain more of their culture of origin, as well as a lesser preference for the adoption of the host culture. In areas considered public, the natives prefer immigrants to adopt more of their host culture and less of their culture of origin. This study supports the theoretical predictions proposed by RAEM regarding the order of the socio-cultural areas, both in the preferences of maintenance and adoption, in both groups of natives studied (those who evaluate Romanians and those who evaluate Ecuadorians).
\end{abstract}

Keywords: acculturation, relative acculturation extended model, Rasch model, item response theory. 


\section{Introducción}

El estudio del proceso de aculturación es especialmente relevante en las sociedades desarrolladas, donde el fenómeno migratorio se ha convertido en un factor estructural y permanente (p.e., Retortillo, 2009). El estudio de la aculturación ha sido muy prolífico en el campo de las ciencias sociales, donde ha sido abordado desde diferentes perspectivas como la antropológica, sociológica y psicológica (p.e., Lakey, 2003; Martín, 2017). Desde una perspectiva psicológica, Berry (1990) entiende la aculturación como un "proceso mediante el cual las personas cambian, siendo influidas por el contacto con otra cultura y participando en los cambios generales de su propia cultura” (p. 460). Las transformaciones culturales inherentes al proceso de aculturación repercuten en la identidad, la actitud, los valores y los comportamientos de los inmigrantes y autóctonos que interaccionan, aunque más intensamente en los primeros (Berry, 1997; Bourhis et al., 1997).

Berry et al. (1989) proponen un modelo bidimensional con dos dimensiones independientes: el mantenimiento de la identidad cultural y las costumbres de su sociedad de origen, y la búsqueda ofomento de las relaciones con la sociedad de acogida, si es que la consideran valiosas. Estas dos dimensiones se pueden combinar para dar lugar a un modelo donde se representan las cuatro posibles opciones de aculturación que ponen de manifiesto los inmigrantes: Integración, donde se mantiene su identidad cultural de origen mientras que se produce relación y apertura con los grupos de la sociedad de acogida; Asimilación, si existe un deseo de abandono de la identidad de la cultura de origen a la vez que se orientan hacia la sociedad de acogida; Separación, caracterizada por mantener la identidad y tradiciones de la sociedad de origen, sin tener relación con la sociedad de acogida; y, Marginación, es el resultado de la pérdida de contacto cultural o psicológico del grupo minoritario con la sociedad de origen como con la sociedad de acogida. Estas dos últimas se denominan segregación y exclusión, respectivamente, cuando esta situación es provocada por el grupo dominante.

En España, las ideas de Berry y sus colaboradores, junto con otros modelos y trabajos que extendían el estudio de la aculturación a otras variables psicosociales relacionadas (p.e., el Modelo Interactivo de Aculturación de Bourhis et al., 1997; los trabajos de Piontkowski et al., 2002), dieron origen al Modelo Ampliado de Aculturación Relativa (MAAR; Navas et al., 2004, 2005). Este modelo, adaptado al contexto social español (Navas et al., 2010), además de recoger las aportaciones que otros autores y estudios ya apuntaban, ha proporcionado otras contribuciones novedosas. En primer lugar, el MAAR considera conjuntamente las opciones de aculturación de la población autóctona 
y de inmigrantes (Bourhis et al., 1997), ya que dependiendo de las opciones por las que opten ambos grupos puede llevar a diferentes tipos de relaciones intergrupales. En segundo lugar, atiende al origen etnocultural de los inmigrantes, ya que dependiendo del grupo considerado puede diferir el proceso de aculturación (Bourhis et al., 1997; Piontowski y Florack, 1995). En tercer lugar, el MAAR trata de comprobar la influencia de predicción y modulación de diferentes variables psicosociales y sociodemográficas sobre las preferencias de aculturación mantenidas por los autóctonos y los inmigrantes. En cuarto lugar, el MAAR apuesta por diferenciar entre las preferencias (plano ideal), opción que escogerían en cada caso si la elección pudiera ser libre, y estrategias o percepciones (plano real) de aculturación, o lo que es lo mismo, qué elección llevan a cabo los inmigrantes o que perciben los autóctonos que estos hacen. En último lugar, el MAAR puntualiza que el proceso de aculturación es complejo y relativo, puesto que se pueden adoptar diferentes opciones de aculturación al mismo tiempo dependiendo del ámbito sociocultural que se considere (Navas y Rojas, 2010).

Esta última puntualización se considera una de las aportaciones fundamentales de este modelo, ya que no solo divide la realidad sociocultural en distintos ámbitos, sino que lleva a cabo la operacionalización de los mismos (Navas y Rojas, 2010). En sus inicios el MAAR distinguía siete ámbitos de aculturación, desde los más públicos (más periféricos a la cultura), como serían el ámbito político -sistema político y de gobierno-, el laboral procedimientos de trabajo- y el económico -hábitos de consumo y economía familiar-, pasando por los ámbitos intermedios como el ámbito social- relaciones sociales y amistades-, hasta aquellos que son denominados el "núcleo duro" de la cultura, es decir, los más privados, como serían el ámbito familiar, religioso y formas de pensar -principios y valores-. Posteriormente, tras considerar los resultados obtenidos en investigaciones realizadas en Andalucía (p.e., Navas et al., 2004, 2006a, 2006b, 2007), el MAAR se ha reestructurado, ampliando a ocho los ámbitos socioculturales: desde los más públicos (político y de gobierno, bienestar social, formas de trabajar), pasando por los centrales (hábitos de consumo y economía doméstica, relaciones sociales), hasta los más privados (relaciones familiares, creencias y prácticas religiosas y valores). En conclusión, el MAAR considera el proceso de aculturación como la síntesis relativa y selectiva o adaptación que realiza una persona entre su cultura de origen y la cultura de acogida en cada uno de los ámbitos mencionados anteriormente, tomando o rechazando los componentes de ambas culturas con distinta magnitud según el ámbito (Navas y Rojas, 2010).

En España, el MAAR ha sido ampliamente aplicado para estudiar el proceso de aculturación de inmigrantes de diferentes orígenes (p.e., Navas et al., 2006a, 2007, 2010; Ordóñez-Carrasco et al., 2020; Rojas et al., 2014). Además, a pesar de haber surgido para 
el contexto español, ha tenido una gran repercusión internacional, ya que ha sido adaptado y aplicado en diferentes países como Canadá (Dupuis y Safdar, 2010), USA (Buckingham, 2018; Thelamour, 2017, Italia (López-Rodríguez et al., 2014), Polonia (Szydłowska, 2019), entre otros.

El MAAR establece un marco conceptual donde se propone la hipótesis de que los ámbitos socioculturales pueden ser divididos y ordenados (figura 1) en función de lo que prefieren mantener y/o adoptar de las costumbres de la sociedad de origen o de la sociedad de acogida (en el caso de los inmigrantes) y en función de lo que prefiere que los inmigrantes mantengan y/o adopten de su cultura de origen o de la cultura de acogida (en el caso de los autóctonos hacia los inmigrantes). En la publicación original del modelo (figura 1) los ámbitos aparecen ordenados uno a uno (Navas y Rojas, 2010) y así han sido tratados en otros trabajos (p.e., Navas et al., 2010). No obstante, trabajos posteriores (p.e., Rojas et al., 2014) han agrupado las puntuaciones de los ámbitos públicos (sistema político y de gobierno, sistema de bienestar social, formas de trabajar), intermedios (hábitos de consumo y economía doméstica, relaciones sociales) $\mathrm{y}$ privados (relaciones familiares, creencias y prácticas religiosas y valores). La hipótesis propuesta por el MAAR sobre el orden jerárquico de los ocho ámbitos y la agrupación de estos (públicos, intermedios y privados) puede y deber ser estudiada con el fin de mostrar evidencia empírica de estos supuestos teóricos.

La aproximación tradicional para la medición mediante escalas de medición (p.e., la Teoría Clásica de los Test) utiliza puntuaciones medias o puntuaciones totales del conjunto de ítems del test y asumen que todos los elementos contribuyen por igual al mismo constructo subyacente y esto puede ser problemático para los ámbitos del MAAR, ya que probablemente algunos ámbitos contribuyan más que otros en la aculturación psicológica. Sin embargo, los modelos basados en la TRI emplean la probabilidad de que una persona dé una respuesta concreta según su nivel habilidad en la variable latente subyacente, donde cada ítem tiene su propio peso para la medida del constructo. En cierta medida, la TRI es análoga a la regresión logística, que es ampliamente utilizada en ciencias biomédicas y psicosociales. La utilización de modelos de medida basados en la teoría de respuesta al ítem (TRI) nos permiten contrastar las hipótesis sobre el orden jerárquico de los ámbitos y la agrupación de estos (públicos, privados, intermedios), especialmente utilizando los mapas de variables. 


\section{Figura 1. Jerarquía de ámbitos socioculturales del MAAR}

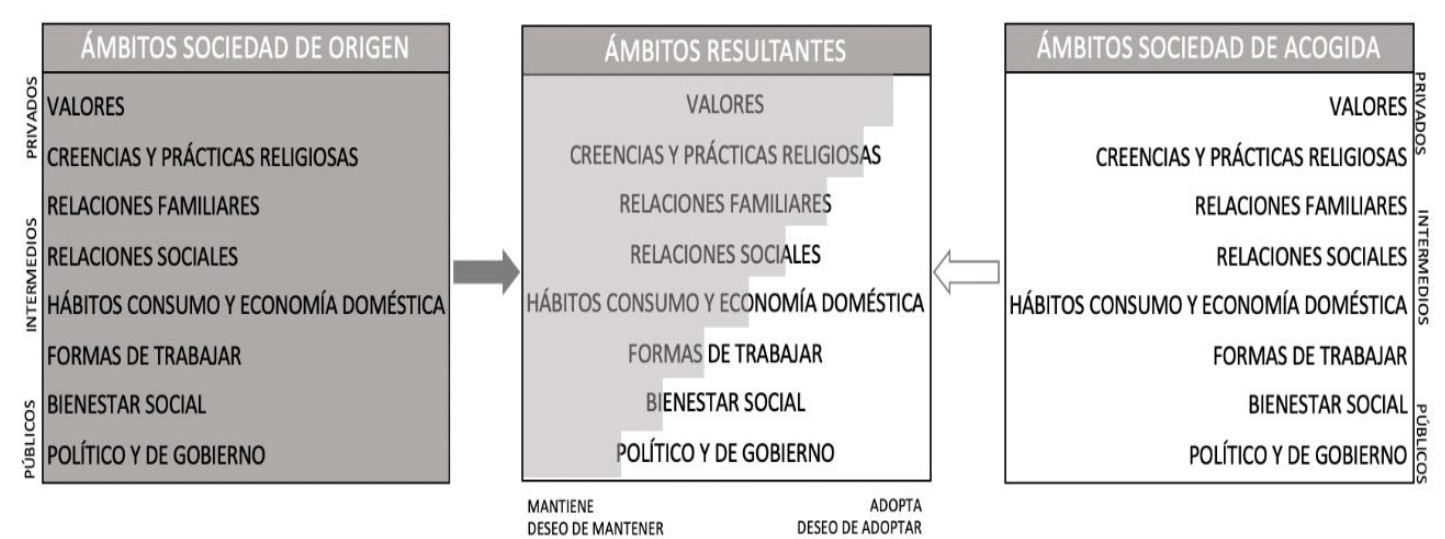

Fuente: Navas y Rojas (2010)

Los modelos basados en la TRI permiten la realización de una representación gráfica del constructo como un mapa de la variable (mapas de personas e ítems), donde se localizan conjuntamente personas e ítems en la misma variable latente que representa el constructo que se mide (Engelhard, 2013). Una implementación de estos mapas de variables se podría realizar mediante el empleo de los modelos de Rasch (p.e., Masters y Wright, 1984).

El objetivo de este estudio es aportar evidencias empíricas del modelo teórico de los ámbitos de aculturación propuestos por el Modelo de Ampliado de Aculturación Relativa (MAAR) a partir de las medidas de preferencias de mantenimiento y adopción cultural aplicando la TRI. Concretamente, se llevará a cabo un estudio a partir de la realización de mapas de variables aplicando un modelo de Rasch para respuestas politómicas, el Modelo de Escalas de Clasificación (Andrich, 1978a, 1978b; Masters, 1980), para ambas escalas (i.e., adopción y mantenimiento) y en distintos grupos (autóctonos que evalúan a rumanos y autóctonos que evalúan a ecuatorianos), con el fin de observar si se replican las estructuras teóricas de los ámbitos. De forma que esperamos encontrar una estructura ordenada para los ítems de los distintos ámbitos desde los más públicos (político y de gobierno, bienestar social, formas de trabajar), pasando por los intermedios (hábitos de consumo y economía doméstica, relaciones sociales), hasta los más privados (relaciones familiares, creencias y prácticas religiosas y valores), y dicha estructura va a tomar diferente forma dependiendo de si se considera la escala de mantenimiento (los autóctonos preferirán que mantengan los ámbitos más privados que los más públicos) o la de adopción (los autóctonos preferirán que adopten los ámbitos más públicos que los más privados). 


\section{Método}

\subsection{Participantes}

Este estudio se llevó a cabo mediante una encuesta en la que participaron 922 personas autóctonas. Concretamente, 493 respondían teniendo como referencia al grupo de inmigrantes ecuatorianos -grupo AE-, y 499 respondían a las preguntas de un cuestionario teniendo como referencia al grupo de inmigrantes rumanos -grupo AR-. Se realizó un muestreo aleatorio multietápico. En la primera etapa, un equipo de expertos seleccionó, a partir de los datos del censo nacional, 12 municipios que se repartían en cuatro comunidades autónomas del Mediterráneo con las tasas más altas de recepción de inmigrantes de estos orígenes (al menos el 5\% del censo tenía que ser inmigrantes y, de éstos, al menos el $2 \%$ personas inmigrantes de cada uno de estos orígenes sobre el total de la población). En la segunda etapa se estratificó, con una asignación proporcional por sexo y edad y, por último, se seleccionaron rutas aleatorias en cada municipio. El error de muestreo se estimó en $\pm 4,3 \%$ ( $p=q=.5$; nivel de confianza: $95.5 \%$ ) para el total de la muestra de autóctonos que evaluaban a ecuatorianos (AE) y rumanos (AR).

La edad media de la población era superior a los 40 años: 45.1 años para el grupo AE $(D T=16.8)$ y 45.0 años para el grupo AR $(D T=16.8)$. El sexo estuvo equilibrado en todas las muestras (alrededor del 50\%).

\subsection{Variables e instrumentos}

Las variables elegidas para probar el modelo fueron las preferencias de aculturación de los autóctonos, que se midieron a partir de un cuestionario donde además se incluyeron las variables sociodemográficas (p.e., sexo, edad).

Dos escalas se utilizaron para conocer las preferencias de aculturación de los autóctonos sobre inmigrantes rumanos y ecuatorianos: la primera escala (Escala de Preferencia de Mantenimiento) estaba enfocada en conocer las preferencias sobre $e l$ grado de mantenimiento de las costumbres del país de origen del inmigrante, mientras que la segunda escala (Escala de Preferencia de Adopción) indagaba sobre las preferencias sobre el grado de adopción de costumbres de la sociedad de acogida ("Si pudiera elegir, ¿en qué grado le gustaría que las personas ecuatorianas/rumanas que viven aquí mantuvieran las costumbres de su país de origen en los siguientes ámbitos?” y “Si pudiera elegir, ¿̇en qué grado le gustaría que las personas ecuatorianas/rumanas 
que viven aquí adoptaran/practicaran las costumbres españolas en los siguientes ámbitos?", respectivamente). Las dos escalas estaban compuestas por 8 ítems referentes a los distintos ámbitos contemplados por el MAAR (e.g., Rojas et al., 2014), que iban desde los ámbitos más públicos (sistema político y de gobierno -forma en que se eligen los gobiernos, forma en que funcionan, participación política, leyes, etc.-, sistema de bienestar social -educación, sanidad y servicios sociales y - formas de trabajar -ritmo de trabajo, horario, condiciones laborales -p.e., desempleo, eventualidad, etc.-), hasta los ámbitos más privados (relaciones familiares -forma de relacionarse con la pareja, hijosas, las personas mayores de la familia, reparto de roles o funciones, etc.-, creencias $y$ prácticas religiosas -creencias, prácticas y cumplimiento personal de obligaciones o prohibiciones religiosas- $y$ Valores -respeto a las personas mayores, forma de educar a los hijos-as igualdad entre hombres y mujeres, papel de la religión en la vida, etc.-), pasando por los intermedios (hábitos de consumo y economía doméstica -productos que compran, tipo de comida que consumen, economía familiar -p.e., dinero que gastan y ahorran, forma de administrar lo que tienen, etc.- y relaciones sociales -forma de relacionarse, lugares habituales de relación social, uso del tiempo libre y formas de divertirse, etc.-; ver figura 1). En ambas escalas las alternativas de respuesta oscilaban desde 1 (nada) hasta 5 (mucho). A mayor puntuación en la escala de mantenimiento mayor preferencia por parte de los autóctonos a que los ecuatorianos/rumanos mantengan sus costumbres de origen. A mayor puntuación en la escala de adopción mayor preferencia por parte de los autóctonos a que los ecuatorianos/rumanos adopten las costumbres españolas.

\subsection{Procedimiento}

La recogida de datos y administración del cuestionario fue realizada por un grupo de diez encuestadores formados a tal efecto. La selección de los autóctonos se basó en una estrategia para asegurar la aleatoriedad y la representatividad de la muestra, a través de sucesivas etapas. En primer lugar, se establecieron los puntos de muestreo de manera aleatoria en los municipios seleccionados (tres puntos de muestreo en cada uno de ellos). En segundo lugar, se definieron los estratos por sexo y edad establecidos a partir de la información de los padrones disponibles. Finalmente, cada encuestador disponía de una hoja de estratos que contenía los perfiles de las personas a entrevistar, y de un punto de muestreo desde el cual iniciar la localización de los encuestados siguiendo las pautas de construcción de rutas aleatorias para las que se encontraban formados. La participación fue voluntaria. 


\subsection{Análisis de Datos}

Se seleccionó el Modelo de Escalas de Clasificación (MEC; p.e., Andrich, 1978a), un modelo de la familia de Rasch, debido a que las dos escalas empleadas para medir las preferencias de aculturación tenían ítems con opciones de respuestas tipo Likert. El MEC nos permite llevar a cabo mediciones de variables psicosociales mediante ítems con alternativas de respuestas ordenadas similares en todos los ítems. Este modelo estima la probabilidad que tiene una persona de responder a una categoría determinada de un ítem deduciéndola de la diferencia entre la cantidad de constructo que posee la persona en la variable que estamos midiendo y el nivel de intensidad que poseen los ítems que han sido utilizados para medir dicha variable. En los modelos de Rasch la unidad de medida es el "logit", que surge de una transformación logarítmica de los datos observados expresándolos en un nivel de medida de intervalo, donde cualquier distancia entre dos puntos en cualquier localización del constructo son equivalentes. El rango de valores de la escala "logit" fluctúa desde menos a más infinito, pero lo más habitual es utilizar un intervalo de \pm 5 , cuyo valor medio es o y su desviación típica 1.

Para comprobar el grado en que los datos empíricos se ajustan al MEC, se han analizado las diferencias entre los valores observados y esperados en términos de residuos. Concretamente se han calculado los residuales cuadráticos medios (MNSQ) mediante dos índices: INFIT y OUTFIT. El INFIT (índice de ajuste interno) es más sensible a los comportamientos inesperados que afectan a los ítems que están cerca del nivel del constructo que poseen las personas, mientras que el OUTFIT, (índice de ajuste externo) es más sensible a los comportamientos inesperados que afectan a los ítems que se encuentran lejos del nivel del constructo que poseen las personas (p.e., Tristán, 1998). Se consideran valores aceptables para estos estadísticos aquellos que se encuentren en el intervalo 0.6 y 1.4 donde 1 sería el valor ideal (Lunz et al., 1990). También se han calculado los valores de la correlación ítem-total para todos los ítems de ambas escalas en ambas muestras, considerándose que todos deben tener valores mayores de .4 y similares entre sí.

El MEC proporciona información sobre los estadísticos de ajuste, la calibración de los ítems (valor de medida de cada ítem), la medida de las personas, así como indicadores de la precisión de la medida. La medida de las personas y la calibración de los ítems se utilizan conjuntamente mediante los mapas de la variable (o mapa de ítems-personas). Sin embargo, para el objetivo de estudiar la validez de la jerarquía propuesta por el MAAR sólo se utilizarán las localizaciones de los ítems en las variables de mantenimiento y adopción en ambos grupos, en función de su valor de medida (cantidad de constructo 
necesaria para ser respondidos favorablemente). Para ello, se han realizado cuatro diagramas de barras elaborados a partir de estos mapas, dos para la muestra AR y dos para la muestra AE, uno por cada una de las escalas. Para una mejor comprensión de los mapas de ítems se hizo una transformación de las puntuaciones "logits" para quitar decimales y expresarla en una escala con media en 5 y desviación típica de 2 (puntuaciones transformadas $=5+2^{*}$ puntuaciones logits).

Los análisis se realizaron con el software WINSTEPS (Linacre, 2007) y los diagramas de barras de las variables se realizaron con el programa Microsoft Excel.

\section{Resultados}

\subsection{Ajuste de datos al modelo}

En cuanto al estudio del ajuste de los datos al modelo, en la Tabla 1 podemos observar los índices de ajuste de los ítems en la escala de preferencia de mantenimiento. En ella vemos que existe un buen ajuste de todos los ítems tanto interno (INFIT) como externo (OUTFIT) en ambos grupos (autóctonos que evalúan a ecuatorianos -AE- y autóctonos que evalúan a rumanos -AR-), puesto que los valores de los residuales cuadráticos medios oscilan entre o.6 y 1.4. En la misma tabla se indica el ajuste de los ítems en la escala de preferencia de adopción. En este caso también existe un buen ajuste de todos los ítems en ambos grupos, debido a que de nuevo tanto el INFIT como el OUTFIT oscilan todos sus valores entre 0.6 y 1.4. En general, existe un buen ajuste total de los datos al modelo en ambas escalas, ya que tanto los valores medios INFIT como OUTFIT están cercanos a 1, tanto el grupo AE como el grupo AR. Los valores de la correlación ítem-total son similares y adecuados para todos los ítems. Las correlaciones son todas medias-altas y oscilaron entre .62 y .73 para la escala de preferencia de mantenimiento y entre $.65 \mathrm{y}$ .79 para la escala de preferencia de adopción.

Por último, los modelos de Rasch ofrecen un coeficiente de separabilidad, que se puede considerar como el equivalente al coeficiente de fiabilidad. En la escala de preferencia de adopción nos encontramos con un valor mínimo aceptable estimado de fiabilidad de las escalas en AR de 0.70 y en AE de 0.69; mientras que en la escala de mantenimiento el grupo AR ha obtenido valores aceptables de 0.75 y el grupo AE de 0.78. 
Tabla 1. Datos de ajuste ítems-modelo.

\begin{tabular}{|c|c|c|c|c|c|c|c|c|c|c|}
\hline \multicolumn{11}{|c|}{ Escala de preferencia mantenimiento } \\
\hline \multirow[t]{2}{*}{ Ítems } & \multicolumn{3}{|c|}{ Medida } & \multirow{2}{*}{$\begin{array}{c}\text { Error } \\
\text { E } \\
\end{array}$} & \multicolumn{3}{|c|}{ INFIT } & \multirow{2}{*}{$\begin{array}{c}\text { OUTFIT } \\
\text { E } \\
\end{array}$} & \multicolumn{2}{|c|}{$\begin{array}{c}\text { Correlación } \\
\text { ítem-total } \\
\end{array}$} \\
\hline & $\mathbf{R}$ & $\mathbf{E}$ & $\mathbf{R}$ & & $\mathbf{R}$ & $\mathbf{E}$ & $\mathbf{R}$ & & $\mathbf{R}$ & $\mathbf{E}$ \\
\hline$\overline{\text { Sistema político/gobierno }}$ & & & & & & & & & & \\
\hline Sistema de bienestar social & 0.46 & 0.03 & .07 & .08 & .26 & .25 & .42 & .22 & .53 & .63 \\
\hline Laboral & 0.09 & 0.75 & .06 & .08 & .08 & .19 & .17 & .58 & .64 & .62 \\
\hline Hábitos de consumo & 0.57 & 0.15 & .06 & .07 & .06 & .07 & .04 & .05 & .69 & .66 \\
\hline Relaciones sociales & 0.05 & 0.68 & .06 & .07 & .88 & .94 & .91 & .95 & .74 & .68 \\
\hline Relaciones familiares & 0.29 & 0.62 & .06 & .07 & .08 & .90 & .84 & .91 & .75 & .72 \\
\hline Creencias/prácticas religiosas & 0.90 & 1.24 & .06 & .07 & .68 & .86 & .68 & .84 & .82 & .70 \\
\hline 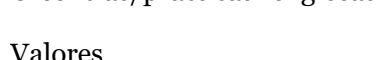 & 1.00 & 1.30 & .06 & .07 & .26 & .98 & .26 & .99 & .74 & .68 \\
\hline Media & 0.89 & 1.08 & .06 & .07 & .91 & .81 & .91 & .82 & .77 & .73 \\
\hline D.T. & 0.00 & 0.00 & .06 & .07 & .00 & .00 & .03 & .04 & & \\
\hline $0^{0.0}$ & 0.89 & 0.31 & .00 & .00 & .19 & .15 & .95 & .23 & & \\
\hline
\end{tabular}

Escala de preferencia adopción

\begin{tabular}{|c|c|c|c|c|c|c|c|c|c|c|}
\hline \multirow[t]{2}{*}{ Ítems } & \multicolumn{3}{|c|}{ Medida } & \multirow{2}{*}{$\begin{array}{c}\text { Error } \\
\mathbf{E}\end{array}$} & \multicolumn{3}{|c|}{ INFIT } & \multirow{2}{*}{$\begin{array}{c}\text { OUTFIT } \\
\mathbf{E}\end{array}$} & \multicolumn{2}{|c|}{$\begin{array}{c}\text { Correlación } \\
\text { ítem-total } \\
\end{array}$} \\
\hline & $\mathbf{R}$ & $\mathbf{E}$ & $\mathbf{R}$ & & $\mathbf{R}$ & $\mathbf{E}$ & $\mathbf{R}$ & & $\mathbf{R}$ & $\mathbf{E}$ \\
\hline Sistema político/gobierno & & & & & & & & & & \\
\hline & 0.65 & 0.80 & .07 & .08 & .33 & .10 & .37 & .17 & .55 & .68 \\
\hline & 0.94 & 1.01 & .07 & .08 & .91 & .08 & .88 & .13 & .60 & .65 \\
\hline & 0.82 & 0.85 & .07 & .08 & .93 & .97 & .89 & .01 & .61 & .69 \\
\hline 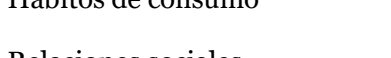 & 0.36 & 0.63 & .06 & .08 & .85 & .91 & .88 & .91 & .70 & .77 \\
\hline & 0.09 & 0.02 & .07 & .08 & .89 & .86 & .91 & .86 & .67 & .77 \\
\hline Creencias/prácticas religiosas & 0.43 & 0.73 & .06 & .08 & .70 & .90 & .70 & .93 & .76 & .79 \\
\hline & 0.52 & 0.43 & .06 & .08 & .38 & .02 & .41 & .04 & .70 & .78 \\
\hline Media & 0.19 & 0.11 & .07 & .08 & .01 & .09 & .03 & .07 & .68 & .74 \\
\hline D.T. & 0.00 & 0.00 & .07 & .08 & .00 & .99 & .01 & .01 & & \\
\hline & 0.76 & 0.30 & .00 & .00 & .22 & .09 & .24 & .10 & & \\
\hline
\end{tabular}

Para obtener evidencias de validez del modelo teórico de los ámbitos de aculturación propuestos por el MAAR es necesario contemplar de manera global cómo se distribuyen empíricamente los ítems según el valor de escala que poseen, tanto de preferencias de mantenimiento como de adopción. Para ello, a partir de los mapas de las variables de ambas escalas se han realizado cuatro gráficas, dos por grupo (AR y AE). En las dos primeras aparecen los ítems que representan a cada ámbito sociocultural que propone el MAAR graduados en función del peso que posee cada ámbito (ítem) en la medida de las preferencias de mantenimiento y de adopción (valor de escala) obtenido en cada 
muestra. En las dos siguientes, aparecen los ámbitos agrupados por públicos, intermedios y privados y graduados en función del peso que posee cada agrupación de ámbitos en la medida de las preferencias de adopción y de mantenimiento (valor de escala promedio) obtenido en cada muestra.

Si analizamos la gráfica de preferencias de mantenimiento vemos que, en general, los ítems (ámbitos) siguen la estructura propuesta por el MAAR, es decir, por un lado, los ítems (ámbitos) que requieren menor valor de constructo para ser respondidos favorablemente (ítems más fáciles) son creencias y prácticas religiosas, relaciones familiares y valores, que son los ítems representantes de los ámbitos privados (figura 2). Mientras que, los ítems (ámbitos) que requieren mayor cantidad de constructo para ser respondidos favorablemente (ítems más difíciles) son bienestar social, formas de trabajar y sistema político y de gobierno, que son los ítems representantes de los ámbitos públicos. Tanto en el grupo AR como en el grupo AE los ítems con mayor valor de constructo son bienestar social, sistema político y de gobierno y formas de trabajar, mientras que los que poseen menor valor de constructo son los ámbitos de creencias $y$ prácticas religiosas, valores y relaciones familiares. Los diagramas reflejan una similitud en ambos grupos a la hora de la preferencia de mantener los ámbitos, aunque con ligeras variaciones en los valores de los ítems (ámbitos). Finalmente, los ítems se distribuyen de la misma manera en ambos grupos exceptuando los ámbitos de hábitos de consumo y economía doméstica y relaciones sociales que para el grupo de AE este último tiene mayor valor que en el grupo AR.

Por otro lado, la gráfica de preferencias de adopción, tanto para el grupo AE como en el AR, sigue la propuesta jerárquica establecida por el MAAR, localizando los ítems (ámbitos) de forma inversa a como lo estaban en la preferencia de mantenimiento (figura 2). Por un lado, los ítems (ámbitos) que requieren menor habilidad para ser respondidos favorablemente (ítems más fáciles) son bienestar social, laboral y sistema político, que son los ítems representantes de los ámbitos públicos. Mientras que, los ítems (ámbitos) que requieren mayor habilidad para ser respondidos favorablemente (ítems más difíciles) son hábitos de consumo, relaciones familiares y religión, que son los ítems representantes de los ámbitos privados. Como bien reflejan los diagramas, en la escala de preferencias de adopción, el ordenamiento de los ámbitos es casi invariante y acorde al MAAR tanto para el grupo AE como el grupo AR. La única excepción está en los ítems de los ámbitos valores y relaciones sociales, que para el grupo AE tiene mayor valor las relaciones sociales y para el grupo AR tiene mayor valor los valores. 
La gráfica de preferencia de mantenimiento agrupadas por ámbitos públicos, intermedias y privadas, ajustan perfectamente a las predicciones del MAAR (figura 2). Las preferencias por que mantengan los ámbitos privados es la que menores valores muestran, siendo los valores mayores para los ámbitos públicos. Igualmente ocurre con la gráfica de preferencia de adopción respecto a lo pronosticado con el MAAR. En este caso, en sentido contrario a los valores de mantenimiento (figura 2). El ajuste en mantenimiento aparece más pronunciado que en adopción.

\section{Figura 2. Diagrama de barras realizado a partir de los mapas de las variables (ámbitos socioculturales del MAAR) agrupados y desagrupados por grupos AE y AR (promedios y barras de error).}

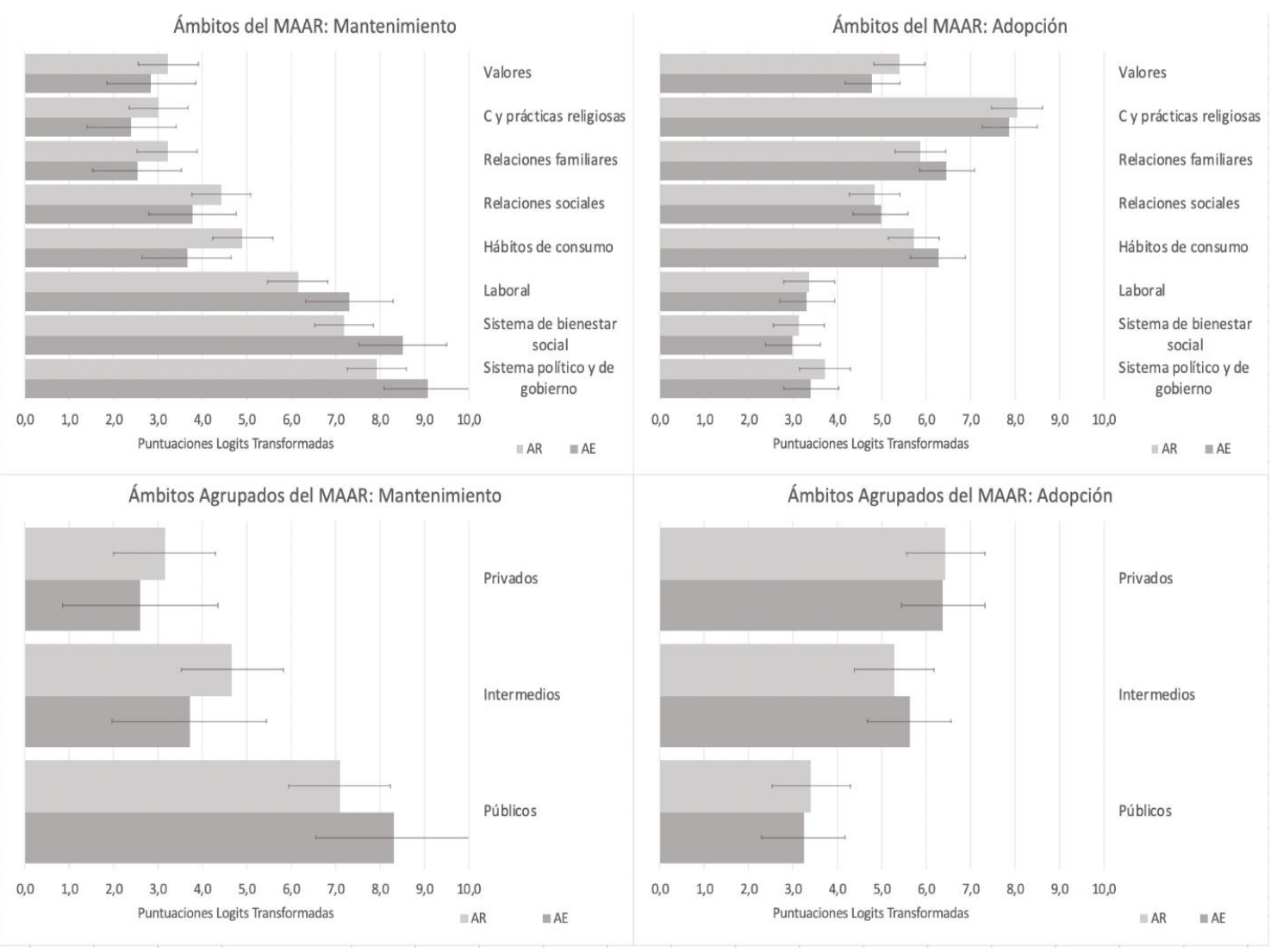

\section{Discusión y conclusiones}

Este trabajo ha tenido como objetivo aportar evidencias empíricas del modelo teórico de los ámbitos de aculturación propuestos por el MAAR mediante la aplicación del MEC, un modelo de TRI perteneciente a la familia de modelos de Rasch. En general, los resultados obtenidos son coherentes con las predicciones teóricas propuestas por el MAAR referentes al orden de los ámbitos tanto en las preferencias de mantenimiento 
como de adopción en ambos grupos de autóctonos estudiados (los que evalúan a rumanos y los que evalúan a ecuatorianos).

Tanto en el grupo $\mathrm{AE}$ como en el grupo $\mathrm{AR}$ vemos que los ámbitos en preferencias de mantenimiento más "fáciles" son los privados - relaciones familiares, religión y valores - es decir, los autóctonos que han evaluado tanto a ecuatorianos como a rumanos prefieren que éstos mantengan los ámbitos privados de su cultura antes que los intermedios y los públicos. En segundo lugar, nos encontramos con los ámbitos des relaciones sociales y bienestar social-, los cuales, a pesar de circunscribirse al ámbito intermedio como se esperaba, intercambian su orden según el grupo: relaciones sociales y hábitos de consumo para $\mathrm{AR}$ y al contrario para el grupo de AE. Por último, tenemos los ámbitos públicos (los ítems más difíciles), lo que quiere decir que los autóctonos prefieren que los inmigrantes (AR y AE) mantengan en menor medida las costumbres de su cultura de origen relacionadas a estos ámbitos. El MAAR predice que los autóctonos, a la hora de evaluar a un grupo de inmigrantes, van a preferir que mantengan los ámbitos privados antes que los públicos debido a que los ámbitos que forman el núcleo duro de la cultura (privados) no entran en contacto con ellos de manera tan directa como los públicos, donde el contacto les afecta directamente.

En cuanto a las preferencias de adopción, en ambos grupos (AE y AR), podemos ver que los ámbitos más "fáciles" son (de menor a mayor dificultad) bienestar, trabajo y político. Este hallazgo es coherente con los resultados obtenidos para las preferencias de mantenimiento, ya que se esperaba que mantuviesen el mismo orden, pero "la dificultad" fuese inversa. Sin embargo, los ámbitos de valores y relaciones sociales mueven su posición. A pesar de que cambien su posición, los valores son coherentes con los esperados para estos ámbitos, que siguen conformando lo que teóricamente llamamos ámbitos privados o "núcleo duro" de la cultura.

Cuando se hace el análisis agrupando los ámbitos en públicos, intermedios y privados (promediando los valores de los ítems que lo integran) tanto el grupo AE como el AR obtienen el mismo orden en mantenimiento y adopción, y acorde con la propuesta teórica del MAAR. Los ámbitos públicos en adopción para ambos grupos (AE y AR) son los más "fáciles” y los ámbitos más privados los más “difíciles”, es decir, los autóctonos prefieren en mayor medida que los inmigrantes adopten las costumbres ligadas al ámbito público de la cultura de acogida y al contrario en el caso de los ámbitos privados. Según estos resultados, podríamos esperar opciones de aculturación de segregación para los ámbitos privados con más facilidad que para los intermedios y para los públicos, y de asimilación para los públicos con más facilidad que para los intermedios o privados, compatibles con 
resultados previos encontrados en otros estudios del MAAR en España (p.e., Navas et al., 2010).

En función de los resultados encontrados se puede afirmar que este estudio muestra evidencias de validez empíricas del Modelo Ampliado de Aculturación Relativa (MAAR), ya que los ámbitos centrales o privados son los que los autóctonos prefieren que los inmigrantes mantengan con más facilidad, mientras que son estos mismos ámbitos los que con mayor dificultar quieren que adopten. Sin embargo, para los ámbitos considerados periféricos o públicos son los que más fácilmente prefieren que adopten los inmigrantes, pero prefieren que mantengan con mayor dificultad.

Todo lo comentado anteriormente ha de interpretarse con cierta cautela, puesto que los resultados obtenidos respecto la distribución de los valores de los ítems (su valor en continuo) es relativamente pequeña en términos de logits.

\section{Financiación}

Este estudio fue financiado por dos proyectos de investigación, uno de la Consejería de Innovación, Ciencia y Empresa de Andalucía (España) en el marco de la Investigación de Excelencia [Po9-SEJ-4657] y otro por el Ministerio de Economía y Industria y Competitividad (PS2016-80123-P). 


\section{Referencias}

Andrich, D. (1978a). A rating formulation for ordered response categories. Psychometrika, 43, 561- 573. http://dx.doi.org/10.1007/BFo2293814

Andrich, D. (1978b). Application of a psychometric rating model to ordered categories which are scored with successive integers. Applied Psychological Measurement, 2(4), 581-594. https://doi.org/10.1177/014662167800200413

Berry, J.W. (1990). Psychology of Acculturation. En J. Berman (Ed.), Cross-cultural perspectives: Nebraska symposium on motivation, (pp. 457-488). University of Nebraska Press. https://doi.org/10.1111/j.1467-6494.2005.00337.x

Berry, J. W. (1997). Immigration, acculturation, and adaptation. Applied Psychology: An International Review, 46, 5-68. https://doi.org/10.1111/j.14640597.1997.tbo1087.x

Berry, J.W., Kim, U., Power, S., Young, M. y Bujaki, M. (1989). Acculturation attitudes in plural societies. Applied Psychology, 38, 185-206. https://doi.org/10.1111/j.1464-0597.1989.tb01208.x

Buckingham, S. L. (2018). When real diverges from ideal: How person-environment fit impacts Latina/o immigrants' acculturation and psychosocial wellbeing across four states. ProQuest Information \& Learning, AAI10253304.

Bourhis, R.Y., Moïse, L.C., Perreault, S. y Senécal, S. (1997). Towards an Interactive Acculturation Model: A social Psychological Approach. International Journal of Psychology, 32(6), 369-386. https://doi.org/10.1080/002075997400629

Dupuis, D.R y Safdar, S. (2010). Terror management and acculturation: Do thoughts of death affect the acculturation attitudes of receiving society members? International Journal of Intercultural Relations, 34(5), 436-451. https://doi.org/10.1016/j.ijintrel.2010.04.006

Engelhard, G., Jr. (2013). Invariant Measurement: Using Rasch Models in the Social, Behavioral, and Health Sciences. Routledge. https://doi.org/10.1007/s11336-0139398-1

Lakey, P. (2003). Acculturation: a Review of the Literature. Intercultural Communication Studies XII, 2, 103-118.

Linacre, J.M. (2007). Winstep (computer program and manual). Chicago: Mesa Press. López-Rodríguez, L., Bottura, B., Navas, M. y Mancini, T. (2014). Acculturation strategies and attitudes in immigrant and host adolescents: The RAEM in different national contexts. Psicologia Sociale, 9(2), 133-157. https://doi.org $10.1482 / 77473 /$ 
Lunz, M.E., Wright, B.D. y Linacre, J.M. (1990). Measuring the impact of judge severity on examination scores. Applied Measurement in Education, 3(4), 331-345. https://doi.org/10.1207/s15324818ameo304_3

Martín, R. (2017). Estudios de aculturación en España en la última década. Papeles del Psicólogo, 38(2), 125-134. https://doi.org/10.23923/pap.psicol2017.2826

Masters, G. N. (1980). A Rasch model for rating scales. Dissertation Abstracts International, 41, 215A-216A.

Masters, G. N. y Wright, B. D. (1984). The essential process in a family of measurement models. Psychometrika, 49, 529-544. https://doi.org/10.1007/BFo2302590

Navas, M., García, M.C., Sánchez, J., Rojas, AJ, Pumares, P. y Fernández, JS (2005). Modelo extendido de aculturación relativa (RAEM): nuevas contribuciones con respecto al estudio de la aculturación. International Journal of Intercultural Relations, 29 (1), 21-37. https://doi.org/10.1016/j.ijintrel.2006.08.002

Navas, M.S., García, M.C. y Rojas, A.J. (2006a). Acculturation strategies and attitudes of African immigrants in the South of Spain: Between reality and hope. Cross Cultural Research, 4O(4), 331-351. https://doi.org/10.1177/1069397105283405

Navas, M.S., García, M.C., Rojas, A.J., Pumares, P. y Cuadrado, I. (2006b). Actitudes de aculturación y prejuicio: la perspectiva de autóctonos e inmigrantes. Psicothema, 18(2), 187-193.

Navas, M. S., Pumares, P., Sánchez-Miranda, J., García, M.C., Rojas, A.J., Cuadrado, I., Asensio, M. y Fernández-Prados, J.S. (2004). Estrategias y actitudes de aculturación: la perspectiva de los inmigrantes y de los autóctonos en Almería. Dirección General de Coordinación de Políticas Migratorias. Consejería de Gobernación. Junta de Andalucía.

Navas, M. y Rojas, A.J. (2010). Aplicación del modelo ampliado de Aculturación relativa (MAAR) a nuevos colectivos de inmigrantes en Andalucía: rumanos y ecuatorianos. Junta de Andalucía.

Navas, M., Rojas, A.J., García, M.C. y Pumares, P. (2007). Estrategias y actitudes de aculturación según el modelo extendido de aculturación relativa (RAEM): las perspectivas de los nativos versus los inmigrantes. International Journal of Intercultural Relations, 31(1), 67-86.

Navas, M., Rojas, A.J., Pumares, P., Lozano, O.M. y Cuadrado, I. (2010). Perfiles de aculturación según el modelo ampliado de aculturación Relativa: Autóctonos, Inmigrantes rumanos y ecuatorianos. Revista De Psicología Social, 25(3), 295312. https://doi.org/10.1177/1069397105283405 
Ordoñez, J., Blanc, A., Navas, M.S. \& Rojas, A.J. (2020). Acculturation preferences of Spaniards towards Middle Eastern and Syrian refugees and their relationship with the stereotypical dimension of morality / Preferencias de aculturación de españoles hacia refugiados sirios y de Oriente Medio y su relación con la dimensión estereotípica de moralidad. International Journal of Social Psychology, 35(1), 936. https://doi.org/10.108o/o2134748.2019.1682291

Piontkowsky, U. y Florack, A. (1995). Attitudes toward acculturation from the dominant group's point of view. Comunicación presentada al VI Congreso de Europeo de Psicología, Atenas, julio.

Piontkowsky, U., Rohmann, A. y Florack, A. (2002). Concordance of acculturation attitudes and perceived threat. Group Processes Intergroup Relations, 5(3), 221232. https://doi.org/10.1177/1368430202005003003

Retortillo, A.A. (2009). Evolución de los modelos psicológicos de aculturación en Norteamérica y en Europa: de la unidimensionalidad a la bidimensionalidad en el tratamiento de la inmigración. Revista de Historia de la Psicología, 3o(1), 73-86.

Rojas, A.J., Navas, M., Sayans-Jiménez, P. y Cuadrado, I. (2014). Acculturation Preference Profiles of Spaniards and Romanian Immigrants: The Role of Prejudice and Public and Private Acculturation Areas. The Journal of Social Psychology, 54(4), 339-351. https://doi.org/10.1080/00224545.2014.903223

Szydłowska, P. (2019). Polish Child Migrants - Psychological Perspective. Prace Historyczne, 146(3), 649-665. https://doi.org/10.4467/20844069PH.19.037.10391

Thelamour, B. (2017). Applying the Relative Acculturation Extended Model to Examine Black Americans' Perspectives on African Immigrant Acculturation. Journal of $\begin{array}{lll}\text { Cross-Cultural } \quad \text { Psychology, } & \text { 1457-1471. }\end{array}$ https://doi.org/10.1177/0022022117730614

Tristán, A. (1998). Análisis de Rasch para todos: una guía simplificada para evaluadores educativos. México: CENEVAL. 